\title{
Observaciones sobre uso y función de arte- factos en Sein und Zeit de M. Heidegger
}

\section{Observations on the use and function of artefacts in M. Heidegger's Sein und Zeit}

\author{
DIEGO PARENTE \\ Conicet - Universidad de Mar del Plata, Argentina.
}

Recibido: 04-05-2007 Aprobado definitivamente: 27-08-2007

RESUMEN

El objetivo del presente artículo es indagar las particularidades del problema de uso y función de artefactos técnicos en el marco de Sein und Zeit. En un primer momento se reconstruye el criterio heideggeriano de interpretación de funciones en objetos técnicos por medio de una identificación de dos niveles de «comprensión». En segundo término se discute el problema concerniente al vínculo entre el sustrato material natural y el útil en cuanto a su estatuto ontológico. Por último se muestra cómo, más allá de la prioridad que otorga a la Umsicht como actividad desocultante, Heidegger indica cierto tipo de constricciones en los artefactos (tanto en su diseño como en su comprensibilidad) que limitarían su «flexibilidad interpretativa».

\section{PALABRAS CLAVE \\ HEIDEGGER , USO, FUNCIÓN, ARTEFACTO TÉCNICO}

\begin{abstract}
This paper intends to investigate some peculiarities of problem of use and function of technical artefacts in Heidegger's Sein und Zeit. First it reconstructs Heideggerian criterion for interpreting functions in technical objects by means of an identification of two levels of «understanding». Then it discusses the problem concerning the link between the natural material substratum and the tool in their ontological aspect. Finally this article shows how Heidegger indicates some constrictions in artefacts (related to their design and comprehensibility) which would limit their «interpretative flexibility».
\end{abstract}

KEYWORDS

HEIDEGGER, USE, FUNCTION, TECHNICAL ARTEFACT

(C) Contrastes. Revista Internacional de Filosofía, vol. XIII (2008), pp. 37-59. ISSN: 1136-4076

Licenciatura de Filosofía, Universidad de Málaga, Facultad de Filosofía y Letras

Campus de Teatinos, E-29071 Málaga (España) 
Si bien HeidegGer no elabora en Sein und Zeit una reflexión sobre la técnica en sentido ontológico como la que orientaría sus meditaciones a partir de la década del treinta, es indudable que entrega una serie de especificaciones sobre los prágmata y su relación con el Dasein. ${ }^{1}$ Como es sabido, tales reflexiones adquieren especial protagonismo en los parágrafos $\$ 15$ y $\$ 16$ de dicha obra («Das Sein des in der Umwelt begegnenden Seienden» y «Die am innerweltlich Seienden sich meldende Weltmäßigkeit der Umwelt»), precisamente allí donde se aborda el problema de la circunmundaneidad (Umweltlichkeit) y la mundaneidad en general (Weltlichkeit).

En estos parágrafos Heidegger ofrece algunas nociones que pueden ser interpretadas como puntos de partida para comprender nuestro vínculo con los útiles, más específicamente para establecer algunas ideas acerca de las funciones de los artefactos y los límites de su interpretación. ${ }^{2}$ Ciertamente estos últimos constituyen tópicos centrales en el debate contemporáneo en filosofía de la técnica. El problema específico instalado en el seno de dicho debate concierne a la posibilidad de descubrir una función inmanente o propia en los artefactos, tarea que implica pensar las condiciones para la asignación de una función en diversos contextos de uso.

$\mathrm{Al}$ igual que en el análisis del significado de una obra literaria, la intentio auctoris, la intentio operis y la intentio receptoris pueden ser pensadas como tres dimensiones de análisis del objeto técnico a la hora de determinar su "paraqué' o referencia teleológica. Estas tres dimensiones aluden a distintos aspectos involucrados en la interpretación de artefactos y en la determinación de su pertenencia a una clase. ${ }^{3} \mathrm{Si}$ bien cada uno de estos abordajes admite matices y variantes de diverso tipo, se puede afirmar esquemáticamente que la intentio auctoris enfatiza que la función propia del artefacto se halla en los contenidos de conciencia del inventor; la intentio operis sugiere que se encuentra en su propio sustrato material (diseño), mientras que la intentio receptoris postula

1 Seguimos aquí el criterio propuesto por Jorge Rivera en su traducción de Sein und Zeit. A fin de evitar las connotaciones de «ser-ahí" (presentes en la célebre traducción de J. Gaos), se conserva el término original 'Dasein' para comprender exclusivamente la referencia a la existencia humana.

2 Las temáticas y el vocabulario que conforman el debate contemporáneo en filosofía de la técnica, especialmente en el ámbito anglosajón, muestran claramente la relevancia de las tesis heideggerianas en este campo disciplinar. Véanse, especialmente, Dreyfus, 1995; Ihde, 2002; Feenberg, 2001; Thomson, 2000; Zimmerman, 1990; Borgmann, 2002.

3 Algunos autores, entre ellos Dennett (1990), sugieren que el problema de la identificación de un 'artefacto' como tal y la asignación de sus funciones es apenas un capítulo de un problema hermenéutico más abarcativo: el que interroga acerca de cómo somos capaces de interpretar y otorgar significado a las acciones de otros seres humanos, otros organismos y a textos en general. 
que la función deriva de las interpretaciones de los usuarios. ${ }^{4}$ En el marco de este trabajo se intentará realizar una serie de precisiones acerca del tratamiento heideggeriano ${ }^{5}$ sobre los útiles con el objetivo de explicitar de qué modo el filósofo alemán opera entre las tres dimensiones de análisis mencionadas y en qué sentido postula cierto tipo de constricciones para la interpretación de artefactos.

\section{LA INTERPRETACIÓN DE ÚTILES EN LOS PARÁGRAFOS $§ 15$ Y $\$ 16$ \\ DE SEIN UND ZEIT}

En lo que sigue se intentará reconstruir las líneas fundamentales presentadas por Heidegger en los parágrafos $\$ 15$ y $\$ 16$ de Sein und Zeit en relación con el tópico elegido. Específicamente, se pretende mostrar de qué manera el filósofo alemán indica una serie de pautas que configuran un criterio fenomenológico para determinar si un útil ha sido apropiado de manera genuina o auténtica. Tal como se argumentará, dicho criterio puede sintetizarse del siguiente modo: cuanto más «desaparece» el útil y menos se percata el agente de su uso, más auténticamente el Dasein se ha apropiado de él.

En $\$ 15$ Heidegger señala que el Dasein se encuentra siempre ocupado en el complejo remisional de los útiles (Zeuge). «Estar en el mundo» significa «absorberse atemática y circunspectivamente en las remisiones constitutivas del estar-a-la-mano del todo de útiles» (1998: 103), es decir, estar inmerso en el trato con $\pi \varrho \alpha ́ \gamma \mu \alpha \tau \alpha$ (prágmata). De tal manera el modo inmediato del trato entre Dasein y ente intramundano no es el conocer puramente aprehensor, sino el ocuparse que manipula y utiliza, el cual tiene su «conocimiento». En el trato cotidiano con los útiles predomina entonces la Umsicht (circunspección), ${ }^{6}$ no homologable a la visión teorética de un sujeto que se «representa» objetos.

En el trato pueden encontrarse los útiles para escribir, los útiles para coser, los útiles para trabajar, los útiles para viajar, los útiles para medir. Es necesario determinar

4 Thomasson (2003) ha desarrollado una teoría histórico-intencional centrada en la intentio auctoris. Grint y Woolgar (1992) y el constructivismo social de Bijker y Pinch (1987) pueden ser considerados como las principales expresiones teóricas de la intentio receptoris. Para una perspectiva crítica sobre esta clasificación, véase Lawler, 2003.

5 Cabe destacar que, a fin de enriquecer y complejizar la discusión heideggeriana tal como aparece en Sein und Zeit, será necesario recurrir a otros textos previos importantes de la década del ' 20 (directamente conectados con la obra de 1927) y a sus reflexiones sobre la obra de arte realizadas en la década siguiente.

6 A diferencia de la Hinsicht (mirada contemplativa), la denominación Umsicht remite aquí a un trato interesado por el mundo, cercano al saber-hacer que opera en la vida diaria. 
el modo de ser de los útiles, y esto habrá de hacerse tomando como hilo conductor la previa delimitación de lo que hace del útil un útil, de la 'pragmaticidad'. Un útil no «es», en rigor, jamás. Al ser del útil le pertenece siempre y cada vez un todo de útiles, en el que el útil puede ser el útil que él es. Esencialmente, el útil es «algo para...». Las distintas maneras del «para-algo», tales como la utilidad, la capacidad para contribuir a, la empleabilidad, la manejabilidad, constituyen una totalidad de útiles. En la estructura del «para-algo» hay una remisión de algo hacia algo. (1998: 96).

De este modo, el martillo se incorpora en el uso, sin ser apropiado primariamente como «objeto», sino como parte de un conjunto de útiles (zapatos, clavos, etc) en un sistema de asignaciones. Heidegger insiste en que los útiles no se hallan aislados, sino que se manifiestan al Dasein como miembros de un contexto (Zeugganzheit), es decir, una totalidad de herramientas, implementos, máquinas, materiales, energías y otros items. Esto implica que el uso de un útil está dirigido -y necesariamente refiere- a otro útil. En la experiencia ordinaria no hallamos items singulares o autosuficientes. Más bien, el útil se encuentra siempre inmerso en un complejo remisional y su uso situado es anterior al sólo mirar las cosas. Es evidente que -en tal contexto- resulta imposible identificar cierto objeto en cuanto a su funcionalidad sin establecer su dependencia en relación con ciertos nexos de sentido, nexos que remiten tanto a un horizonte más amplio de objetos como a una estructura de acciones intencionales posibles. Esto significa que a fin de identificar un objeto en cuanto «artefacto» se requiere introducirlo en el marco de una actividad determinada. ${ }^{7}$

La praxis humana involucrada en la manipulación de útiles no es, según Heidegger, una mera cognición perceptual, sino un tipo de relación familiar, un cierto interés activo que trata las cosas y las pone en uso. El Dasein se encuentra con los útiles, en primer lugar, a través de la manipulación. En cuanto menos se atienda a la «cosa-martillo», y más se la use, más originaria o primordial será nuestra relación con él. En tal sentido, el reconocer algo como «martillo» no significa adjudicarle una serie de propiedades físicas particulares. Más bien, a fin de comprender el martillo (lo cual implica saber usarlo apropiadamente) resulta necesario adscribir relaciones hacia otros útiles y actividades, tales como golpear una madera, sacar clavos, etc. En su argumentación Heidegger alude insistentemente a la «transparencia» del instrumento: cuando estamos usando un útil, éste tiende a desaparecer, no nos percatamos de que tenga características. El bastón de un individuo ciego deviene «transparente» si es que ha sido apropiado de la manera más genuina (es decir, cuando no es vivenciado como un conjunto de propiedades en cuanto a peso, textura, etc., sino como una simple extensión 
del sentido del tacto). Por otra parte, también la comprensión del propio Dasein se vuelve transparente en este estar sumergido en la Zeugganzheit.

Ahora bien, en este marco dispuesto por Heidegger en los parágrafos mencionados puede afirmarse que no hay comprensión auténtica de los útiles a través de la mera contemplación desapegada sino sólo a través del uso. Un artefacto técnico es definido por aquello para lo cual es usado normalmente por un usuario normal y en una cultura donde tal objeto tiene una función establecida. ${ }^{8}$ En términos generales, el reconocimiento de algo como útil, es decir, el reconocimiento de un cierto para-qué, supone una pre-comprensión activa. Es así que el uso efectivo en el marco de una serie de tareas es esencial para una comprensión originaria. Un breve pero esclarecedor pasaje de Der Ursprung des Kunstwerk resume perfectamente esta idea ya delineada en las páginas de Sein und Zeit:

[...] las botas campesinas las lleva la labradora cuando trabaja en el campo y sólo en ese momento son precisamente lo que son. Lo son tanto más cuanto menos piensa la labradora en sus botas durante su trabajo, cuando ni siquiera las mira ni las siente. La labradora se sostiene sobre sus botas y anda con ellas. Así es como dichas botas sirven realmente para algo. Es en este proceso de utilización del utensilio cuando debemos toparnos verdaderamente con el carácter de utensilio. (1997: 26).

En segundo lugar, como bien indica Hubert Dreyfus, también se puede tener una comprensión derivada o positiva de los útiles con el sólo estar familiarizado con su función normal. ${ }^{9}$ En este sentido sabemos qué es un rastrillo o unos anteojos sin necesidad de haberlos utilizado previamente. En estos casos, la identificación de su función, o la asignación de un cierto «para-qué», requiere una percatación temática deliberada. A través de esta última, el Dasein descontextualiza su objeto revelando sus atributos libres de contexto -por ejemplo, las «propiedades» particulares de un martillo. La mera «objetividad»-en cuanto presencia o estar-ahí (Vorhandenheit)- es un modo de ser derivado resultante de dicha actividad.

En su interpretación de Heidegger, Dreyfus señala que al encargarnos atentamente de las cosas, primero notamos que tienen ciertas funciones. Por ejemplo, al decidir qué tipo de manija instalar en una puerta o qué hacer cuando se atasca, la circunspección descubre para qué sirve la manija, pero sólo la comprende plenamente al usarla. Afirma Dreyfus: «Ya hemos usado algo como algo [manijas para abrir puertas] pero ahora lo vemos como algo para 
algo [una manija como un «algo» destinado a abrir puertas]». ${ }^{10}$ De tal modo, la identificación de un objeto en cuanto a su funcionalidad requiere una labor de descripción temática, mientras que el saber usarlo implica sólo una percatación atemática, una familiaridad práctica, el dominio de un cierto know-how -es decir, un tipo de saber que si bien puede ser sistematizado en un conjunto de reglas o representaciones no consiste estrictamente en tal conjunto. ${ }^{11}$

Ahora bien, en sus reflexiones de los años treinta, más exactamente en Der Ursprung des Kunstwerk, Heidegger destaca una tercera modalidad de interpretación del útil ciertamente conectada con la anterior: la que se realiza a través de la obra de arte. Esta última, al menos cuando resulta ser figurativa o intenta reproducir un útil de manera deliberada, es otra vía de descubrimiento que no consiste en su uso efectivo. En tal sentido, en la obra de arte se nos muestra cómo la verdad del útil puede ser desocultada por una descontextualización o distanciamiento del trato circunspectivo familiar. Cuando Van Gogh representa un par de zapatos en su pintura, logra mostrar lo que ellos verdaderamente son, alcanzando aquello que no puede captarse en la Umsicht de quien los porta o instrumentaliza. Sin embargo, aquello que se desoculta aquí no es sólo el para-qué singular del zapato en cuanto útil sino el mundo mismo en el que se inserta: el mundo del trabajo (Werkwelt), el mundo del campesino que los usa para determinadas tareas, los distintos significados adheridos a ese esfuerzo, etc. Afirma Heidegger:

Ha sido la obra de arte la que nos ha hecho saber lo que es de verdad un zapato [...] la obra no ha servido únicamente para ilustrar mejor lo que es un utensilio, tal como podría parecer en un principio. Por el contrario, el ser-utensilio del utensilio sólo llega propiamente a la presencia a través de la obra y sólo en ella. (1997: 28-29).

La apropiación realizada por el artista a través de su obra no debe confundirse entonces con la apropiación auténtica del usuario involucrado prácticamente con el útil. Éste descubre al objeto técnico a través del uso; el artista, en cambio, lo descubre mediante una actitud contemplativa que Heidegger conectará posteriormente con el «poetizar». No es casual hallar una explicitación del significado del útil en el marco de una reflexión sobre la obra de arte puesto que, para el filósofo alemán, tanto uno como otra comparten el carácter de Handwerk, esto

10 Dreyfus, 1996: 216.

11 Sin duda es posible imaginar un libro de instrucciones destinado a enseñar el uso apropiado de un determinado útil (por ejemplo, un manual para conducir un automóvil o para manejar una computadora). Pero la Umsicht que activa los usos y los hace efectivos en el marco de una labor particular no puede agotarse en un mero listado de reglas o representaciones, por muy pormenorizado que éste sea. 
es, pertenecen a la esfera de la producción humana en cuanto ambas requieren acción manual. El útil se halla entre la cosa y la obra en la medida en que es «mitad cosa» (en cuanto determinado por la Dingheit) pero también «mitad obra de arte» (por su estatuto de Handwerk), aunque no es autosuficiente como esta última dado que el artefacto requiere de un agente intencional externo para ser puesto en funcionamiento y alcanzar su telos. Mientras que el ser-útil del útil reside en su fiabilidad, la obra de arte -en cambio- no porta ninguna referencia teleológica y en tal sentido se parece a una «cosa espontánea». ${ }^{12}$

En resumen, de acuerdo con lo expuesto anteriormente es posible distinguir algunos trazos de lo que Heidegger podría pensar respecto al acceso cognitivo a los objetos artificiales. En primer lugar, el tipo de conocer que nos permite acceder al modo de ser estos objetos funcionales es de tipo práctico. En segundo término, existen alternativas de descubrimiento de objetos artificiales basadas en una percatación temática, ya sea que ésta se realice a través de un contemplar e identificar funciones, o bien a través de la actividad descontextualizante que caracteriza a la obra de arte.

\section{LA NATURALEZA Y EL PROBLEMA DE LAS CONDICIONES PREVIAS DE LA UMSICHT}

El hecho de que el Dasein comprenda exitosamente las funciones de un útil en tanto las descubre en su trato circunspectivo (Umsicht) no significa que la manifestación efectiva de tales funciones no esté posibilitada, a su vez, por algo distinto a tal trato. De hecho, como se verá en la sección III, Heidegger brinda algunas pistas para pensar cuáles son estas condiciones o requisitos previos sin necesidad de recurrir a conceptos fuertemente vinculados a la tradición tales como el de «sustancia», esto es, sin necesidad de postular la existencia de un sustrato independiente del Dasein sumergido en la actividad de desocultación. En una primera aproximación, por tanto, no cabría postular una sustancia ensí situada más allá de la actividad circunspectiva en la medida en que, según Heidegger, el «algo en cuanto algo» sólo aparece a través del trato (Umgang).

Podría pensarse, sin embargo, que en cuanto una misma cosa puede funcionar como teléfono y pisapapeles (dos «formas» simultáneas), la cosa como es en sí misma no puede ser ni lo uno ni lo otro. En esta concepción tradicional de la sustancia, como sugiere Dreyfus, se supone que debe haber algo que «subyace a estas dos perspectivas subjetivas y a sus respectivos predicados-de-uso, y ésa debe ser la cosa como sustancia, independiente de nuestras proyecciones subjetivas» (1996: 75). Tal lectura supone que los atributos de uso de una cosa -su función como útil-son relativos al interés, de modo que no se hallan en la cosa

12 Heidegger, 1997: 26 y ss. 
misma, sino que más bien son exteriores a ella. Esta última posición conduce a una interrogación acerca del estatuto ontológico de ambas instancias: ¿qué es lo más básico en el nivel ontológico: los útiles o el de-qué material (Woraus) en base al cual aquéllos pueden funcionar efectivamente? En varios pasajes de Sein und Zeit, Heidegger rechaza la alternativa de pensar que primero se experimenta una cosa que está-puramente-ahí, y que luego se la entiende, por ejemplo, en cuanto «puerta» o en cuanto «casa».

El modo de ser de este ente [el útil] es el estar a la mano. Pero el estar a la mano no debe ser entendido como mero carácter aprehensivo, como si al «ente» inmediatamente compareciente se le endosasen luego tales «aspectos» y de esta manera se «colorease subjetivamente» una materia cósmica, que estaría primero presente en sí [...] esto va en contra del sentido ontológico del conocimiento, acerca del cual hemos hecho ver que es un modo fundado del estar-en-el-mundo. (1998: 99). ${ }^{13}$

Heidegger refuerza esta posición cuando afirma que la interpretación tiene una función aperiente, es decir, ella

no arroja cierto «significado» sobre un nudo ente que está-ahí, ni lo reviste con un valor, sino que lo comparece dentro del mundo, ya tiene siempre, en cuanto tal, una condición respectiva abierta en la comprensión del mundo, y esta condición queda expuesta por medio de la interpretación. (1998: 173).

De tal modo, el ver sólo una mera «cosa física» constituiría para Heidegger una forma privativa de ver que -en sí misma- presupone el trato cotidiano y que a su vez se manifiesta en la explicitación. ${ }^{14} \mathrm{Su}$ análisis del uso del viento por el campesino (1998: 81) y el ejemplo de la mesa (2000: 114-117) se dirigen a demostrar que las cosas no se encuentran como entidades presentes aisladas a las que agregamos predicados de función de manera independiente. Para que el campesino use el viento como útil, el viento ya se debe manifestar como teniendo cabida dentro del mundo cotidiano, no como presencia de conjunto de moléculas de aire. De manera similar, la mesa no se presenta primariamente

13 Algo similar sostiene Heidegger en Ontología: «Para entender adecuadamente la estructura fenoménica de la presencia es importante ver de consuno el para-qué en cuanto aquí originario y próximo, y no explicarlo como algo posteriormente hallado, como si fuera finalidad que luego se le hubiera adherido» (2000: 125).

$14 \mathrm{Al}$ respecto Heidegger afirma: «El nada-más-que-tener-ante-sí una cosa se da en el puro quedarse mirando esa cosa en cuanto ya-no-comprenderla. Esta percepción carente de 'en cuanto' es una privación del simple ver comprensor, no más originaria que éste, sino derivada de él [...] toda percepción de un útil a la mano es comprensora-interpretante y deja comparecer circunspectivamente algo en cuanto algo» (1998: 173). 
como una cosa espacial-material a la que, en una instancia posterior, se le asignarían ciertas predicaciones de valor (el estar «bien construida», el «servir para apoyar cosas», etc.). Presuponer que las «cosas de valor» tienen siempre como sustrato el ser una «cosa natural» implica, según Heidegger, una descripción «errónea» del mundo cotidiano.

Ahora bien, la relación entre naturaleza y útiles en cuanto a su prioridad ontológica se ve complejizada en cuanto se presta atención al hecho de que los útiles que comparecen en el mundo del trabajo (Werkwelt), en el trato circunspectivo, remiten necesariamente a materiales «naturales». Escribe Heidegger:

En la obra hay también una remisión a «materiales». Ella está necesitada del cuero, del hilo, de los clavos, etc. El cuero, a su vez, es producido a partir de las pieles [...] Martillo, alicate, clavo, remiten por sí mismos al acero, hierro, mineral, piedra, madera -están hechos de todo eso. Por medio del útil está descubierta también la «naturaleza», y lo está a la luz de los productos naturales. (1998: 98)

Si bien este pasaje no fuerza a admitir que Heidegger piense en términos de «sustancias» naturales, al menos es necesario conceder que sí concibe requisitos previos en los entes que comparecen como «útiles». Estos últimos están conformados de cierta manera (no sólo material sino formal, es decir, a nivel de su diseño) y sin esta conformación adecuada ni siquiera sería posible afirmar legítimamente que el Dasein se encuentra en trato con «útiles». En otras palabras, sería inadecuado sostener que el Dasein se encuentra en trato con un «martillo» en sentido estricto si el ente correspondiente no estuviera conformado por ciertas propiedades indispensables para cumplir apropiadamente prestaciones tales como golpear clavos, ejercer presión, etc. En tal caso no podría afirmarse que tal ente es un «martillo» ya que no cuenta con las prestaciones elementales asignadas convencionalmente a dicho útil -es decir, el tipo de prestaciones en base a las cuales los martillos son identificados como tales. Un caso distinto sería si en tal ente es posible identificar propiedades funcionales que lo insertan en la clase «martillo» pero, debido a diversas causas - por ejemplo, el hallarse roto- no cumple con la servicialidad supuesta. Pero el hecho de que, en un determinado momento, un útil no pueda cumplir con su función supuesta no es argumento suficiente para excluirlo de una determinada clase artificial. ${ }^{15}$

Lo cierto es que la presencia de un material natural (el de-qué) debe ser considerada como una condición de posibilidad para el funcionamiento de los útiles en la medida en que el apropiado desenvolverse de éstos supone ciertos poderes causales anclados en la naturaleza. ${ }^{16}$ Tal reconocimiento no significa,

$15 \mathrm{Al}$ respecto, véase Vega, 2007.

16 Un breve pasaje de Prolegómenos para una historia del concepto de tiempo (lec- 
sin embargo, que la naturaleza posea una prioridad ontológica ya que en esa dimensión de análisis un útil es en relación al contexto reticular en el que se inserta y se fundamenta en nexos de sentido relacionados con actividades emprendidas por el Dasein en su cotidianeidad. En otras palabras, sin una comprensión originaria de nuestro trato con el entorno, sin Zuhandensein, no habría siquiera posibilidad de descontextualizar un útil en sus propiedades funcionales ni de justificar su operatividad por medio de una explicación óntica de sus poderes causales. ${ }^{17}$

En definitiva, resulta evidente que la referencia heideggeriana a los «materiales naturales» que conforman los distintos útiles pone en primer plano la cuestión del papel que juegan las propiedades funcionales en la definición de la naturaleza de un cierto artefacto técnico. Por otra parte, esta remisión abre el camino para una interrogación sobre las constricciones que de diverso modo actúan sobre los artefactos técnicos, tema que se abordará en la siguiente sección.

III. LAS CONSTRICCIONES MATERIALES DE LOS ARTEFACTOS Y LOS CONDICIONAMIENTOS DE LA INTERPRETACIÓN DE SUS FUNCIONES

De acuerdo con lo explicitado anteriormente, queda claro que una descripción de los artefactos en cuanto «sustancias» a las que se agregarían «funciones de uso» en el transcurso de una determinada labor está fuera del proyecto ontológico de Sein und Zeit. Sin embargo, tal consideración no impide hallar en el discurso heideggeriano sobre los útiles una indicación de cierto tipo de constricciones en su estructura.

Si bien Heidegger tematiza la problemática de la «función» del útil, no ofrece ninguna consideración explícita que permita dar una respuesta coheren-

ciones dictadas en 1925) enfatiza esta cuestión: «[...] como las propias remisiones halladas en el análisis del mundo del trabajo nos llevan al final a reconocer y a caracterizar como estrato fundamental de lo real el mundo (de la) naturaleza, vemos que la presencia (Präsenz) mundana verdaderamente primordial no es lo puesto al cuidado en toda ocupación, sino la realidad de la naturaleza. Y esta conclusión, a lo que parece, no hay manera de eludirla» (2006: 249-250). De todos modos, Heidegger entiende que si bien es posible brindar una explicación (óntica) acerca del funcionamiento de un útil mediante la naturaleza, ésta -comprendida en cuanto objetividad-no puede explicar la mundaneidad.

17 Heidegger distingue estos dos tipos de explicaciones en $\S 16$ : «Con mucha frecuencia nos referimos de un modo ónticamente enfático a este en-sí del ser y, desde un punto de vista fenoménico, con razón. Pero este recurso óntico no basta para satisfacer la necesidad del enunciado ontológico presuntamente establecido en él [...] el ser-en-sí del ente intramundano sólo es captable ontológicamente sobre la base del fenómeno del mundo» (1998: 103). 
te frente a una de las cuestiones centrales del actual debate en filosofía de la técnica, a saber, la «flexibilidad interpretativa» ${ }^{18}$ de los artefactos. ¿Existe una ilimitada gama de apropiaciones de objetos técnicos? ¿Cuáles son los límites para interpretar «funciones» en dichos artefactos? En el contexto de Sein und Zeit no es posible hallar argumentos que permitan establecer si puede haber una pluralidad de apropiaciones verdaderamente «genuinas» respecto de un mismo objeto técnico. Como se ha señalado, un útil es apropiado de modo genuino si «desaparece» en el trato circunspectivo del Dasein, es decir, si no hay una percatación temática deliberada de sus propiedades. Pero los ejemplos mencionados en los parágrafos $\$ 15$ y $\$ 16$ (el martillo, los zapatos, el reloj, entre otros) refieren siempre a usos «propios» cuya interpretación no generaría conflictos. Dice Heidegger:

La obra que se quiere producir, y que es el para qué del martillo, cepillo y aguja, tiene, por su parte, el modo de ser del útil. El zapato es para llevarlo (útil-zapato), el reloj, para ver la hora. La obra que comparece con prioridad en el trato en la ocupación -la que se elabora- hace comparecer conjuntamente, en el uso al que por esencia está destinada, el para-qué de $s u$ empleabilidad. (1998: 97).

Es evidente que estos ejemplos no discuten sino que, más bien, reafirman el carácter «propio» de los útiles mencionados. Esta orientación tiende a ignorar la posibilidad de imaginar otros usos alternativos capaces de descubrir la misma estructura material de manera «auténtica» y de hacer comparecer a un útil dentro del mundo. De este modo, el interrogante que no llega a ser enunciado de modo explícito es el de la determinación de la interpretabilidad del útil -por ejemplo, no se llega a responder si el uso del zapato como matamoscas es un tipo de apropiación auténtica o si se trata, más bien, de una desviada o impropia. Si el Dasein, en su dirección interpretante-comprensora, está capacitado para desocultar entes en cuanto «útiles» y para determinar incondicionalmente su significado, entonces no parece haber obstáculos a priori para tratar al zapato en términos de «útil para matar moscas»o, ciertamente con más dificultad, como «útil para realizar cálculos matemáticos». Tal conclusión es forzosa excepto que la interpretación de funciones se vea limitada por algún componente externo a la propia actividad de desocultación en la que se ve involucrado el agente.

18 En base a esta noción, el constructivismo social de Bijker y Pinch (1987) pretende señalar el carácter constitutivamente social de los artefactos. En esta perspectiva, tanto el diseño como la producción y el uso de objetos funcionales es el resultado de las interpretaciones de los grupos sociales relevantes, no la materialización de un sentido previo determinado por un inventor singular. 
De tal modo -y ciertamente vinculado al tema abordado en sección [2]- aparece la cuestión de si la interpretación de funciones técnicas está limitada por alguna clase de instancia, ya sea material, cognitiva, cultural o de algún otro tipo. En este sentido, es posible hallar en el discurso de Sein und Zeit una serie de referencias significativas a [a] algunas constricciones materiales -corporizadas e internalizadas en la estructura de los propios útiles-que, en cierto modo, limitan sus usos, y [b] la idea de Vorhabe (o «haber previo») que condicionaría en sentido fuerte las posibles interpretaciones sobre sus funciones propias.

\section{1. CONSTRICCIONES MATERIALES}

En una serie de reflexiones sobre las vinculaciones entre diseño, usuario y entorno, Heidegger señala que la estructura del útil en cuanto objeto de diseño no es arbitraria. Ella descansa, al menos, sobre dos tipos de condicionamiento claramente diferenciables. Por un lado, el útil indica -a través de su diseño- una corporalidad subyacente. Todo artefacto se encuentra estructurado sobre nuestra organización anatómica, tanto sobre sus características como sobre sus límites. Específicamente, Heidegger indica que en el diseño se manifiesta el cuerpo del agente involucrado:

La obra producida no sólo remite al para-qué de su empleo y al de-qué de su composición; en condiciones artesanales simples, ella remite, además, al portador y usuario. La obra se hace a su medida; él 'está' presente al surgir la obra. (1998: 98).

El mango de un cuchillo o la manija de una puerta remiten a esta corporalidad subyacente. No sería legítimo considerar como «útil» a un objeto que careciera de tales disposiciones. De hecho, sin tal adecuación a lo somático, se manifestaría sencillamente como un ente que «está ahí», como mera presencia. El diseño de un determinado útil exige, entonces, que el agente involucrado en su manipulación realice una cierta clase de operaciones definidas de manera precisa: tanto la manija de la puerta como el mango del cuchillo deberán ser manipulados de un cierto modo si es que se pretende alcanzar una acción eficaz. A su vez resulta evidente que la estructura material del útil también limita el campo de operaciones posibles, seleccionando aquellas eficientes y exitosas, y descartando el resto (por ejemplo, la alternativa de manejar el cuchillo tomándolo por su hoja). ${ }^{19}$

19 Aquí resulta imprescindible destacar que, en Sein und Zeit, el análisis heideggeriano toma como modelo el útil premoderno, es decir, aquel caracterizado por: [a] conservar un nexo morfológico con algún órgano o prestación congénita del cuerpo humano (brazo, pierna, manos, etc.) y [b] requerir de acción humana efectiva para su funcionamiento. De allí que queden 
Por otra parte, el diseño no sólo se ve constreñido por estos aspectos somáticos, sino también por las propiedades del entorno en el cual el artefacto está destinado a funcionar. Esta configuración se funda en una búsqueda de acoplamiento eficaz con el ambiente. Escribe Heidegger:

En los caminos, carreteras, puentes y edificios, la ocupación descubre la naturaleza en determinada dirección. Un andén techado tiene en cuenta el mal tiempo; las instalaciones del alumbrado público, la oscuridad, es decir, la particular mudanza de la presencia y ausencia de la claridad del día, la 'posición del sol'. En los relojes se tiene en cuenta una determinada constelación del sistema sideral. [...] En el uso de ese útil tan inmediata e inadvertidamente a la mano que es el reloj se encuentra también a la mano la naturaleza del mundo circundante. (1998: 98-99).

Aquello que el filósofo alemán describe en este pasaje es una característica imprescindible del «buen diseño». Éste, como sostiene H. Simon, requiere tener en cuenta la estructura interna del artefacto y el medio en el que actúa en la medida en que el funcionamiento eficaz del útil depende de la articulación entre estas dos instancias. ${ }^{20}$ Pese a evitar el término «diseño» y sus implicaciones, Heidegger da a entender que las sinergias del ambiente son descubiertas y aprovechadas a través de una instancia deliberada de producción de artefactos -actividad que, a su vez, debe tener en cuenta las Naturmächte, debe considerarlas a fin de hacerlas fructíferas. El diseño se dirige, entonces, a controlar todo aquello que el entorno tiene de inseguro, de caótico e imprevisible para enfatizar, en su lugar, la «confiabilidad» y «servicialidad» de los útiles. Aquí vale recordar que, en el marco de Sein und Zeit, una de las modulaciones bajo las cuales se descubre la naturaleza es la Naturmacht, la fuerza natural que acecha nuestro conjunto de útiles y su ocupación. Tal peligro, en cierto modo, es previsto por el Dasein en la decisiva instancia de configuración de sus útiles.

Adecuación a la corporalidad humana y adaptación eficiente al ambiente son, por tanto, constricciones de todo artefacto técnico. Tales constricciones son tenidas en cuenta en el diseño y, en tal sentido, afectan a los materiales de que se componen los útiles. Heidegger se refiere a este vínculo entre materia y útil cuando afirma que este último consiste en una materia comprendida dentro de una forma. En los útiles

al margen aquellas máquinas o servomecanismos cuya estructura no está concebida desde la imagen del cuerpo y aquellos mecanismos en cuyo despliegue el factor humano sólo juega un papel secundario -tales como un sistema de montaje de piezas en el sentido taylorista.

20 Simon concibe al artefacto como punto de unión entre un medio interior (su organización en cuanto tal) y un medio exterior (las proximidades donde actúa). Sólo cuando el medio interior es adecuado al exterior, el artefacto puede cumplir la finalidad para la cual está destinado (H. Simon, 1973: 21 y ss.). 
[...] la forma determina el ordenamiento de la materia. Y no sólo esto, sino también hasta el género y la elección de la misma: impermeable para el cántaro, suficientemente dura para el hacha, firme pero flexible para los zapatos. Además, esta combinación de forma y materia ya viene dispuesta de antemano dependiendo del uso al que se vayan a destinar el cántaro, el hacha o los zapatos. Dicha utilidad nunca se le atribuye ni impone con posterioridad a entes del tipo del cántaro, el hacha y los zapatos. Pero tampoco es alguna suerte de finalidad colgada en algún lugar por encima de ellos. (1997: 21-22).

La utilidad determina y sirve de fundamento a la conformación y a la elección de la materia del útil. Esta constricción es fundamental ya que señala una condición material imprescindible para que el útil pueda efectivamente aparecer como tal en su faz óntica. De tal manera, en esta perspectiva las posibilidades de uso no parecen ser ilimitadas puesto que ellas se encuentran necesariamente restringidas por las legalidades naturales y su «presión selectiva». El carácter no-arbitrario del diseño conduce a pensar que al menos una serie finita de alternativas de uso ya ha sido prevista. Tal serie revistiría, en cierto modo, una prioridad práxica respecto de otros usos potenciales. Esta prioridad debe ser comprendida en un sentido peculiar: la referencia a los materiales hace pensar que no todos los útiles están capacitados para realizar una serie infinita de labores; por el contrario, la identificación de su «en cuanto» está posibilitada por el hecho de que responden de mejor manera a la realización de ciertas tareas antes que a otras. Más allá de sus diferencias, tales características de diseño vinculadas al aspecto somático del usuario y al entorno en el cual se inserta parecen ser constitutivas de todo útil.

Aquí cabe preguntarse por qué Heidegger no profundiza la cuestión del diseño en los parágrafos de Sein und Zeit mencionados más atrás y por qué aborda dicha temática sólo de manera lateral en su reflexión sobre la obra de arte. La marginalidad del problema del diseño de útiles dentro de su obra de 1927 puede comprenderse, fundamentalmente, a partir de dos motivos. En primer término, porque la determinación de la Zuhandenheit en su nivel ontológico requiere poner en primer plano la actividad de «ocupación» del Dasein sin aludir estrictamente a los contenidos de tal praxis como si ellos tuvieran una existencia objetiva independiente. En segundo término, la elipsis sobre dicha temática está dirigida a evitar las implicancias representacionalistas, voluntaristas y, en resumen, metafísicas, del término en cuestión. Por otra parte, puede conjeturarse que en su escrito sobre la obra de arte Heidegger no está preocupado por elaborar una caracterización óntica de los útiles ni de sus prerrequisitos. Más bien, su objetivo allí es ofrecer una definición de la coseidad (Dingheit) de la cosa que logre escapar de la clave sustancialista que prima en el análisis filosófico tradicional. Lo cierto es que, a pesar de este carácter lateral y tácito 
de la cuestión del diseño, Heidegger no puede evitar referirse al estatuto de la materia que, una vez que entra en relación con el Dasein, se presta a ser conformada de modo intencional.

\section{2. LA IDEA DE VORHABE COMO CONDICIONANTE DE LA INTERPRETACIÓN DE FUNCIONES}

¿Es la interpretación de funciones una actividad absolutamente desligada de todo prejuicio o precomprensión? En caso de que la respuesta sea negativa, ¿cuáles son las condiciones culturales previas imprescindibles para la interpretación de funciones en artefactos técnicos? En la sección \$32 de Sein und Zeit Heidegger ofrece una distinción entre Verstehen y Auslegung que puede orientarse a fin de justificar la siguiente idea: el funcionamiento conjunto de una totalidad de útiles (Zeugganzheit) no se apoya en un vacío ahistórico sino que, más bien, se sitúa sobre precomprensiones de carácter histórico-cultural.

[La interpretación sobre la Zuhandenheit] se funda siempre en un haber previo (Vorhabe) [...]. La interpretación se funda siempre en una manera previa de ver (Vorsicht) que «recorta» lo dado en el haber previo hacia una determinada interpretabilidad [...]. La interpretación de algo en cuanto algo está esencialmente fundada en el haber previo, en la manera previa de ver y en la manera de entender previa. La interpretación no es jamás una aprehensión, sin supuestos, de algo dado. (1998: 173-174).

En rigor, la idea de un «haber previo» $(\text { Vorhabe })^{21}$ vinculado con toda interpretación ya aparece delineada en los escritos heideggerianos de inicios de la década del '20, más específicamente en sus Phänomenologische Interpretationen zu Aristoteles (1922) y en Ontologie (1923). En la primera de estas obras Heidegger afirma:

La vida fáctica se mueve en todo momento en un determinado estado de interpretación heredado, revisado o elaborado de nuevo. La circunspección ofrece a la vida un mundo ya interpretado de acuerdo con aquellas perspectivas en las que el mundo comparece y aguarda en calidad de objeto de la preocupación, en las que el mundo es puesto como problema y buscado como refugio. Estas perspectivas, que en la mayoría de los casos no están explícitamente disponibles y por las que

21 En Ontología, Heidegger define el término Vorhabe del siguiente modo: «El mirar a algo y el determinar, activo en ese mirar, lo que está a la vista, en cuanto actuación que lo configura, supone tener ya de antemano lo que se va a mirar en cuanto ente que es así y asá. Lo que de esa manera se tiene de antemano en todo acceso a lo ente y trato con lo ente lo denominaremos haber previo (Vorhabe)» (2000: 104). Acerca del significado de este término en la obra temprana de Heidegger, véase Segura Peraita, 2002: 16 y ss. 
la vida fáctica se desliza por la fuerza del hábito mucho más que por la fuerza de una apropiación expresa, trazan las vías de realización por las que discurre la actividad del cuidado. (2002: 37).

Ahora bien, si se pone en relación esta idea de Vorhabe con el uso de artefactos podría pensarse que las labores -y, por tanto, toda Umsicht relacionada con ellas- se encuentran condicionadas por una serie de reglas que indican los usos «propios» o «normales» de los útiles. Es evidente que si tales usos no estuvieran de algún modo presupuestos en el trato cirunspectivo, no habría lugar para los distintos tipos de «perturbación» que interrumpen la absorción del Dasein en su complejo de útiles y remite a éstos a un plano de mera presencia objetual (Vorhandenheit). Heidegger habla, en tal sentido, de tres tipos de perturbaciones cuya función es llevar a primer plano la característica de estar-ahí del útil: Auffäligkeit, Aufdringlichkeit y Aufsässigkeit (llamatividad, apremiosidad y rebeldía). En la primera de ellas, el útil se vuelve notorio en cuanto aparece en un cierto no-estar-a-la-mano (1998: 73). En la Aufdringlichkeit, la actividad en curso se ve bloqueada, por lo cual-privados del acceso a lo acostumbrado- debemos actuar de modo deliberado, prestando atención a lo que hacemos. $^{22}$ Por último, la Aufsässigkeit refiere a una situación en la que la ausencia de un componente del todo de útiles puede llevar a un desplazamiento desde lo no a-la-mano a lo presente.

Es claro que tales perturbaciones sólo pueden manifestarse como tales a condición de que exista un trasfondo de habitualidad, esto es, un trato rutinizado y automático con un cierto artefacto. ${ }^{23}$ Este último implica la posesión de un know-how relativo a su uso y una cierta precomprensión culturalmente transmitida acerca de cómo deben interpretarse sus funciones. Estos tres modos de perturbación indican, también, que la especificación de las funciones de un cierto útil en términos de sus prestaciones actuales manifiestas es insuficiente: un bolígrafo con la punta dañada no deja de ser bolígrafo incluso si no cumple aquello para lo cual se supone que está ahí. ${ }^{24}$

Por otra parte, ciertos usos deliberadamente desviados (como el ready-made «Fuente» realizado por Duchamp en 1917) ponen bajo interrogación la cuestión de los usos «propios» y la autenticidad de las apropiaciones de artefactos. Al ser situado como obra de arte en el contexto de un museo, el mingitorio se ve despojado de su carácter de útil, se ve privado intencionalmente de su para-qué

22 Tal deliberación significa realizar una actividad reflexiva de planificación, acción que Heidegger remite al esquema «wenn - so» [si-entonces] (1998: 359).

23 Respecto de esta idea Heidegger argumenta en su Ontología que sólo sobre la base del hábito puede aparecer en la inmediatez del aquí mundano algo así como 'lo inhabitual', aquello que «resulta un estorbo», «es inoportuno», «una molestia», etc. (2000: 127).

$24 \mathrm{Al}$ respecto véase Vega, 2007. 
establecido en el diseño. La estrategia de Duchamp consistió en plasmar un gesto artístico que mostraba la relatividad y la génesis institucional de las divisiones entre obra de arte y objetos funcionales por medio de un desplazamiento del objeto de un contexto a otro. También en este caso resulta evidente que sin la preexistencia de ciertas precomprensiones respecto de los usos «propios» del mingitorio, experimentos estéticos como el del artista francés no habrían resultado exitosos o, al menos, no habrían revestido tanta atención.

\section{Acerca de la inserción cultural de los útiles}

El argumento heideggeriano en torno a la naturaleza de los útiles (Zeuge) muestra una oscilación entre la focalización de sus aspectos funcionales y de sus aspectos culturales. Tal distinción atraviesa la reflexión contemporánea sobre la técnica de una manera tan decisiva que, en verdad, no sería inadecuado dividir dichas reflexiones en base al criterio de cuál de ambos aspectos prioriza su estudio. Por un lado, el artefacto cumple una función, responde a los «paraqué» convencionales ya constituidos $\mathrm{y}$, en este sentido acotado, cabe aplicar el esquema problema/solución. Por otro, el artefacto es portador de mediaciones $\mathrm{y}$, en tal sentido, uno de los principales vectores de cultura. Estas dos facetas sólo resultan discernibles analíticamente, puesto que en el marco de las acciones técnicas concretas, el útil es uno y alberga ambos aspectos de manera conjunta y simultánea. En la literatura contemporánea sobre el tema, el estudio de estos dos aspectos se encuentra generalmente escindido. La filosofía analítica de la técnica se ha ocupado casi exclusivamente de la primera faceta, priorizando el estudio de los componentes de la acción técnica, la relación del conocimiento científico con el conocimiento técnico y el estatuto de los valores internos. La segunda cara, en cambio, ha sido abordada no tanto por filósofos, sino por científicos sociales, siendo objeto de estudios etnográficos de diverso tipo.

Ahora bien, más allá de su manifestación en el marco de una cierta labor, todo objeto técnico porta una referencia teleológica, es decir, indica un para$q u e ́$ hacia el cual se dirige, y según el cual fue fabricado y adaptado. ${ }^{25} \mathrm{Si}$ nos centramos en su funcionalidad es innegable que todo útil se halla ciertamente vinculado a la resolución de problemas y, en tal sentido, resulta juzgable en términos de una acción con objetivos y resultados. De hecho, las especificaciones de esta cara del artefacto hallan su traducción dentro del lenguaje del proyectista, en el que se distingue la etapa del problem-setting (en la que se

25 Heidegger focaliza este aspecto funcional del útil en su noción de Verweisung (remisión). Tugendhat (2002) ha señalado que, en Sein und Zeit, este término resulta confuso en cuanto designa dos fenómenos distintos: la relación medio/fin (el para-algo) y, simultáneamente, la relación de reticularidad entre los útiles dentro de una totalidad -por ejemplo en un taller. 
colocan los términos del problema) y la etapa del problem-solving, durante la cual se organizan los medios para resolverlo. ${ }^{26}$

Sin embargo, desde una perspectiva heideggeriana no sería adecuado pensar en el útil como una simple mediación funcional absolutamente desprovista de contenidos culturales y reductible sin resto al mencionado esquema problema/ solución. En cierto modo, la descripción fenomenológica del Zeug provista en las secciones $\$ 15$ y $\$ 16$ de Sein und Zeit focaliza los rasgos del artefacto que se resisten a ser comprendidos como propiedades de un objeto purificado de elementos culturales. El útil se cuida, es guardado en un sitio particular, se inserta en el marco de una labor realizada colectivamente y significativa para un cierto grupo, y forma parte de un complejo de útiles -los cuales se remiten entre sí. En este sentido es posible afirmar que no hay útiles «aislados»-y, menos aún-funciones en sí mismas. Al mismo tiempo, los artefactos son soportes semióticos, transmiten información -por lo cual cabe hablar de ellos como "portadores» de mediaciones - y plasman valores socialmente aceptados y reconocidos. Todo objeto técnico es investido de valores, los cuales se adhieren a sus funciones $\mathrm{u}$ orientaciones originarias conformando una sola unidad indivisible. ${ }^{27}$ Tales características no se aplican exclusivamente a la tecnología moderna sino también a los conjuntos útiles más primitivos. En rigor, la acción técnica responde a varios criterios simultáneamente: los internos (sintetizables bajo el esquema problema/solución) y los extra-técnicos, esto es, aquellas consideraciones de tipo estético, ético y religioso que juegan un papel no menos relevante que el anterior criterio. ${ }^{28}$ Una acción técnica, entonces, no concluye simplemente con la ejecución del gesto eficaz, sino que se incluye en una red de significados más amplia. Por más que la modernidad haya favorecido el fortalecimiento de una cultura secularizada y haya debilitado los componentes ceremoniales tradicionales, toda acción técnica conserva la referencia a un todo extratécnico.

Es importante destacar que el hecho de que los artefactos porten mediaciones y las funciones sean objeto de interpretación y, por tanto, sean resigni-

26 Al respecto, véase Manzini, 1993: 57 y ss.

27 Un cáliz, por ejemplo, implica la función de recipiente pero su uso simbólico en ciertas ceremonias presupone el reconocimiento de los aspectos estéticos y religiosos que lo constituyen. En este sentido, sería insuficiente representarlo sólo como un objeto destinado a servir como «recipiente». Sobre esta inevitable presencia de valores en el diseño de objetos funcionales, véase Simondon, 1969.

28 Si bien Heidegger piensa prioritariamente en los útiles de las sociedades tradicionales, también los artefactos descartables propios de nuestra contemporaneidad portan significados. En tal sentido, la provisoriedad constitutiva de los útiles descartables no puede ser homologada a las conductas de un primate que abandona un instrumento luego de su uso, puesto que la «descartabilidad» es en sí misma una opción cultural surgida históricamente y no es asignable a nuestra especie en cuanto rasgo biológico. 
ficables, es posible por la preexistencia de un mundo cultural. Este mundo de símbolos compartidos es condición de posibilidad de la comprensión y uso de artefactos, así también como de su fabricación. Siguiendo las implicaciones de esta posición, el «mundo» en el cual se inserta una acción técnica o un artefacto particular es un «mundo cultural», es decir, uno constituido por una red de significados. ${ }^{29}$ Tal dimensión cultural no es hallable en otros animales -ni en los insectos sociales, ni en los variados e ingeniosos usos de herramientas en los chimpancés. En cierto modo podría afirmarse que los organismos que forman parte de protoculturas sólo son capaces de «resolver problemas», es decir, de hallar vías efectivas de menor o mayor grado de ingenio destinadas a solucionar dificultades o superar obstáculos de diverso tipo, partiendo siempre desde estímulos ambientales específicos. Ciertamente los animales son capaces de encontrar aspectos operativamente relevantes para conducir sus acciones en el medio ambiente en cuanto disponen de un nivel básico de instrumentalidad. Su Umwelt está conformado por las impresiones del mundo exterior que le resultan relevantes para sus intereses vitales, no por la totalidad de los elementos del hábitat. ${ }^{30}$ Pero el medio ambiente humano complejiza este Umwelt animal. Desde los tiempos del sapiens sapiens se trata de un entorno artificializado, construido y apropiado simultáneamente en el diseño (primero balbuciente; luego, progresivamente, cada vez más metódico y coordinado intersubjetivamente; finalmente, apoyado en el conocimiento científico).

De tal modo, los humanos -en tanto que seres culturales- agregan la capacidad de «abrir mundos» mediante la acción técnica, comprendiendo heideggerianamente la posibilidad de instaurar nuevas constelaciones de significación y de inteligibilidad. ${ }^{31}$ Precisamente aquello que el filósofo alemán intenta indicar mediante su análisis fenomenológico de los útiles es el hecho de que el Dasein necesariamente habita en el espacio abierto por la técnica, es decir, que no hay «mundo» sin esa orientación instrumentalizadora que caracteriza a la ocupación. Afirmar que todo diseño, construcción y utilización de artefactos porta un «mundo» consigo significa que el objeto técnico involucrado en toda acción remite a un horizonte de posibilidades intencionales dentro de las cuales adquiere sentido. De tal modo, la técnica no se limita a asegurar la supervivencia de la especie, es decir, a ofrecer posibilidades de 'adaptación' biológica, sino que transforma la realidad abriendo nuevos campos de inteligibilidad, radi-

29 Se sigue aquí el concepto semiótico de cultura propuesto por Clifford Geertz (1995): 'cultura' es un sistema ordenado de significados compartidos en términos de los cuales los individuos interpretan su experiencia y guian sus acciones, sentimientos y juicios.

30 Sobre esta noción de Umwelt, véase el influyente trabajo de von Uexküll (1956).

31 Los «mundos» abiertos por la técnica, en este sentido heideggeriano, son campos de significados y prácticas antes que colecciones de objetos. Una defensa de este concepto de «mundo» en clave heideggeriana puede verse en Feenberg, 2003: 98 y ss. 
calmente inaccesibles sin ella. En cuanto componente de la herencia cultural, la técnica humana se inscribe en un proceso de transmisión «horizontal» de información que suele sintetizarse en los mismos artefactos. Éstos transportan no sólo mediaciones ético-estéticas, sino también un know-how, el gesto eficaz que descubre al útil en cuanto útil. En este sentido, los artefactos constituyen un archivo o catálogo de los problemas y soluciones particulares abiertos por una cultura. De allí que una aproximación hermenéutica a los útiles de un cierto grupo humano requiera ser capaz de tomar tales elementos como indicios de un horizonte de inteligibilidad, de un «mundo». ${ }^{32}$

\section{CONSIDERACIONES FINALES}

El presente trabajo ha intentado realizar algunas precisiones sobre las particularidades del problema de uso y función de artefactos técnicos en el marco de Sein und Zeit de Martin Heidegger.

En la sección I se reconstruyó el criterio heideggeriano de interpretación de objetos técnicos por medio de una identificación de dos niveles de «comprensión» de útiles, una originaria (el trato circunspectivo atemático) y otra derivada (la percatación temática de funciones). En este sentido los artefactos se nos manifiestan en la manipulación de acuerdo con un saber práctico resultante de la ocupación del Dasein en el mundo cotidiano. La sección II estuvo dedicada a discutir el problema concerniente al vínculo entre el sustrato material natural y el útil. Allí se revisaron las alternativas sobre el estatuto ontológico de cada uno y las variantes y oscilaciones en el discurso heideggeriano en torno a este tópico. Posteriormente se mostró de qué modo, más allá de la prioridad que otorga a la Umsicht como actividad desocultante, Heidegger indica cierto tipo de constricciones en los artefactos, tanto en su diseño como en su comprensibilidad. Por último, la sección III.2.1. intentó brindar una lectura en clave heideggeriana sobre el mundo cultural como condición de posibilidad de fabricación, uso e interpretación de útiles. Tal tarea requirió aclarar la constitutiva inserción cultural de los artefactos en el marco de una concepción que piensa al hombre habitando en el espacio abierto por su instrumentalidad.

Los argumentos trazados anteriormente podrían sugerir que el análisis fenomenológico heideggeriano opera fundamentalmente en el nivel del intérprete (intentio receptoris) en cuanto pone en primer plano el hecho de que el modo de ser de los útiles es primariamente aquel que se manifiesta en el trato circunspec-

32 El segundo Heidegger atendió especialmente a esta característica al afirmar que ciertos artefactos son capaces de funcionar como coligadores de la Cuaternidad (Geviert). Véanse Heidegger, 1967a y 1967b. 
tivo, y no el encontrarse como meras entidades objetivas dotadas de funciones. Sin embargo, en algunos pasajes que abordan la cuestión del diseño, Heidegger se refiere indirectamente $-y$, por supuesto, evitando una idea de «sustancia» en sentido estricto- al propio sustrato material del artefacto. Allí distingue, como se ha visto, las constricciones somáticas (vinculadas al usuario) y las ambientales (las Naturmächte enfrentadas al útil y acopladas en su estructura). Por otra parte, la captación de una «totalidad de útiles» (Zeugganzheit) y las interpretaciones que ponen a sus partes en funcionamiento no están libres de prejuicio sino que, más bien, se sitúan sobre precomprensiones de carácter histórico-cultural. Tales elementos pueden contribuir a pensar que Heidegger visualiza las propiedades estrictamente técnicas de los artefactos y, en tal sentido, no puede postularse que defienda una posición extrema de «flexibilidad interpretativa». Más bien, da lugar para pensar en ciertos límites (materializados en el diseño) para la interpretación de funciones. En cierto modo esta lectura de Heidegger permite postular la existencia de ciertas restricciones impuestas por la propia estructura del artefacto. Admitir tales restricciones, a su vez, equivale a reconocer que no todas las interpretaciones lógicamente posibles son técnicamente compatibles con un cierto útil.

Por último, es importante destacar que una lectura crítica global sobre el problema de uso y función de artefactos en Sein und Zeit requiere reconocer la deliberada originalidad del vocabulario desplegado en dicha obra. A lo largo de su escrito, Heidegger recurre insistentemente a nuevas designaciones (asignadas al campo ontológico) a fin de tomar distancia de las nociones clásicas utilizadas por la tradición -las cuales permanecerían situadas en el nivel óntico. La utilización de términos entrecomillados tales como «Um-zu» (la estructura del «para-algo»), o de otros como Wozu (el para-qué) y Woraus (el de-qué o composición del útil) indican su preocupación por establecer un nuevo léxico para pensar la instrumentalidad fuera de los conceptos de 'medio' y 'fin'. ${ }^{33} \mathrm{De}$ allí que lo que pretende evitar es un lenguaje centrado en las ideas de un agente intencional que se representa objetos en términos de medios y fines activados por acciones causales -es decir, el entramado conceptual asociado a la ontología tradicional cuyos fundamentos pretende deconstruir.

33 Algunos autores, entre ellos Tugendhat, advierten que este intento heideggeriano de pensar la relación instrumental medio/fin a partir de su noción de «Um-zu» no es, en rigor, una alternativa a la explicación tradicional, sino únicamente «una abreviatura de ésta y remitida a ella» (2002: 250). 


\section{REFERENCIAS BIBLIOGRÁFICAS}

BIJKER, Wiebe, PINCH, Trevor et alii, eds. (1987), The Social Construction of Technological Systems, Cambridge: MIT Press.

BORGMANN, Albert (2002), «Focal things and practices», en DREYFUS, H. y WRATHWALL, M. (eds.), Heidegger reexamined, Vol. 3: Art, Poetry and technology, Nueva York: Routledge.

DENNETT, Daniel (1990), «The Interpretation of Texts, People and Other Artifacts», Philosophy and Phenomenological Research, (L), pp. 177-194.

DREYFUS, Hubert (1995), «Heidegger on gaining a free relation to technology», en FEENBERG, A. y HANNAY, A., comps., The Politics of Knowledge, Bloomington: Indiana University Press.

DREYFUS, Hubert (1996), Ser-en-el-mundo. Comentarios a la división I de Ser y Tiempo de Heidegger, Santiago de Chile: Cuatro Vientos.

FEENBERG, Andrew (2001), «The Ontic and the Ontological in Heidegger's Philosophy of Technology», Inquiry, 43, (4), pp. 445-450.

FEENBERG, Andrew (2003), «Modernity Theory and Technology Studies: Reflections on Bridging the Gap», en MISA, Th. y BREY, P. (eds.), Modernity and Technology, MIT Press.

GEERTZ, Clifford (1995), La interpretación de las culturas, Barcelona: Gedisa.

GRINT, K. y WOOLGAR, S. (1992), «Computer, Guns and Roses: What's Social about being shot?», Science, Technology and Human Values, 17, n 3, pp. 366-380.

HEIDEGGER, Martin (1967a), «Die Frage nach der Technik», Vorträge und Aufsätze, Tübingen: G. Neske.

HEIDEGGER, Martin (1967b), «Bauen, Wohnen, Denken», Vorträge und Aufsätze, Tübingen: G. Neske.

HEIDEGGER, Martin (1997), «El origen de la obra de arte», en Caminos de bosque, trad. H. Cortés y A. Leyte, Madrid: Alianza.

HEIDEGGER, Martin (1998) [ed.orig. 1927], Ser y tiempo, trad. J. Rivera, Santiago de Chile: Editorial Universitaria.

HEIDEGGER, Martin (2000), Ontología. Hermenéutica de la facticidad, trad. J.Aspiunza, Madrid: Alianza.

HEIDEGGER, Martin (2002), Interpretaciones fenomenológicas sobre Aristóteles, trad. J. Adrián Escudero, Madrid: Trotta.

HEIDEGGER, Martin (2006), Prolegómenos para una historia del concepto de tiempo, trad. J. Aspiunza, Madrid: Alianza.

IHDE, Don (2002), Bodies in technology, Londres: University of Minnesota Press.

LAWLER, Diego (2003), «Las funciones técnicas de los artefactos y su encuentro con el constructivismo social en tecnología», CTS Revista Iberoamericana de Ciencia, Tecnología y Sociedad, 1, (1), pp. 27-71.

MANZINI, Ezio (1993), La materia de la invención, Barcelona: CEAC.

SEGURA PERAITA, Carmen (2002), Hermenéutica de la vida humana. En torno al Informe Natorp de M.Heidegger, Madrid: Trotta.

SIMON, Herbert A. (1973) Las ciencias de lo artificial. Barcelona: ATE. 
SIMONDON, Gilbert (1969), Du mode d'existence des objets techniques, Paris: Aubier-Montaigne.

THOMASSON, Amie (2003), «Realism and Human Kinds», Philosophy and Phenomenological Research, LXVII/3, pp. 580-609.

THOMSON, Iain (2000), «From the question concerning technology to the quest for a democratic technology: Heidegger, Marcuse, Feenberg», Inquiry, 43, (2), pp. 203-215.

TUGENDHADT, Ernst (2002), «Dificultades en el análisis heideggeriano del mundo circundante», en Problemas, Barcelona: Gedisa.

UEXKÜLL, Jakob von (1956) [1934], Streifzüge durch die Umwelten von Tieren und Menschen. Bedeutungslehre, Hamburgo: Rowohlt.

VEGA, Jesús (2007), «El conocimiento de los artefactos», manuscrito.

VEGA, Jesús y LAWLER, Diego (2005), «La experiencia del mundo técnico», CTS. Revista Iberoamericana de Ciencia, Tecnología y Sociedad, 2, (5), pp. 67-80.

ZIMMERMAN, Michael (1990), Heidegger's confrontation with modernity: Technology, politics, art, Bloomington: Indiana University Press.

Diego Parente. Doctor en Filosofía (Universidad de Buenos Aires, Argentina). Profesor del Departamento de Filosofía de la Universidad Nacional de Mar del Plata, Argentina.

\section{Publicaciones recientes:}

Márgenes del lenguaje. Metáfora y conocimiento (2002) y co-editor de El legado de Immanuel Kant: actualidad y perspectivas (2004). Ha publicado varios artículos sobre problemáticas de filosofía de la técnica en revistas especializadas nacionales y extranjeras, entre ellos, «Tecnología y Gelassenheit. Heidegger y la apertura de futuros artificiales alternativos», Argumentos de razon técnica, Universidad de Sevilla, no 9, 2006, pp. 37-61 y «Observaciones sobre el concepto de tecnología en el diálogo entre Sloterdijk y Heidegger», Revista LOGOS (México), vol. XXXIV, $n^{\circ}$ 101, año XXXIV, mayo-agosto 2006, pp. 97-114.

Dirección postal:

Mitre 1359, $4^{\circ}$ E, CP 7600, Mar del Plata (Argentina).

Dirección electrónica: dparente@uolsinectis.com.ar 
